\title{
Utilisation of Digital Twin in Representation of a Physical Object, Process, or Service
}

\author{
Rudra Bhanu Satpathy ${ }^{1}$, J.Sunil Gavaskar ${ }^{2}$ \\ ${ }^{1}$ M.Tech,Electrical, Electronics and Communications Engineering, St. Peter's University Chennai. \\ ${ }^{2}$ Assistant Professor, Lord Jegannath College of Engineering \& Technology, Ramanathichenputhur. \\ ${ }^{1}$ rudra@gmail.com, ${ }^{2}$ sun.gavas@gmail.com
}

Abstract - Digital twins are a useful tool for integrating cyber-physical processes in production. In order to attain a new higher degree of efficiency, smart manufacturing services might improve the complete business process and operation method of manufacturing. When intelligent production solutions and smart sensors are combined, product development, production and other operations are anticipated to be drastically altered. The digital twin, when paired mostly with technologies, can enable more accurate industrial scheduling and execution management, resulting in industrial automation. Manufacturers are using the many components of a digital twin as services, as this article explains and illustrates.

Keywords - Digital twins, products, market, product development, internet, Services.

\section{Introduction}

A digital twin is a representation of a real thing, operation, or function in digital format. Jet engines, wind turbines, or perhaps even entire towns can be digital twins. Along with property resources, digital twin technologies may be used to mimic operations to gather data on how they will function in the future. It is computer software that simulates the performance of a product or process using real-world data. Software analytics and internet-of-things technology can be used in conjunction with these applications to improve their output. In this research article a brief overview will be provided on the "utilization of digital twins in representation of a physical object, process, or service".

Aim: The aim of this research study is to evaluate and analyze the impact of digital twins in representation of a real asset, operations and function procedure.

The objectives of this research article are to understand the concept of "Digital twins" and its benefit on the techno arête transactions. To identify the factors that is creating a positive impact on the utilization of "digital twins". To identify the challenges in the inclusion of digital twins that is creating a negative impact on the performance of digital twins and to recommend effective solutions to prevent the negative factors in the utilization of digital twins.

Concept of the digital twins

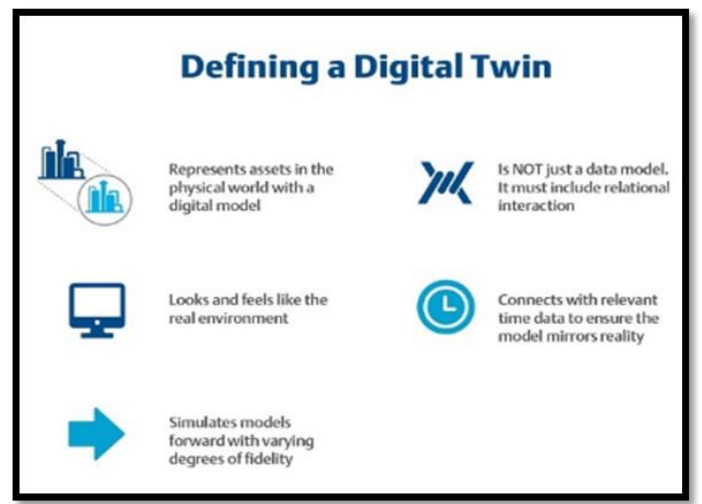

Figure 1: Digital twin

(Source: Qi et al. 2018) 
Virtually depiction of an isolated system that covers its whole lifespan, is refreshed from actual data, and relies on simulations, artificial intelligence and inference to assist in judgment is called a digital twin. As per the words of Qi et al. (2018), digital twins are the digital equivalent to a physical item or process. In 2010, NASA developed the first realistic definition of a digital twin to improve the physical model simulation of spacecraft. To create a digital twin, specialists in applied mathematics or data science must study the physics and operational data of a physical object or system in order to construct a computational formula that replicates the authentic.

\section{Services applications in digital twin}

A resource reservoir and administration framework is used to publish the digital twin services after they have been encapsulated. According to Cimino et al. (2019), hardware services, service operations, image processing, communication services, assisting employees, simulation services, simulation payment systems, etc. are all included in the digital twin services. Additional services include finance, logistical, education, and equipment maintenance. In order to administer services, a task must be submitted to the management platform. It is then broken down into smaller jobs that may be handled by a single service. Quos is used to match supply and demand for manufacturing services in order to pick the optimum solutions (Jones et al. 2020). Services are triggered and integrated to finish the job cooperatively once the service task is made.

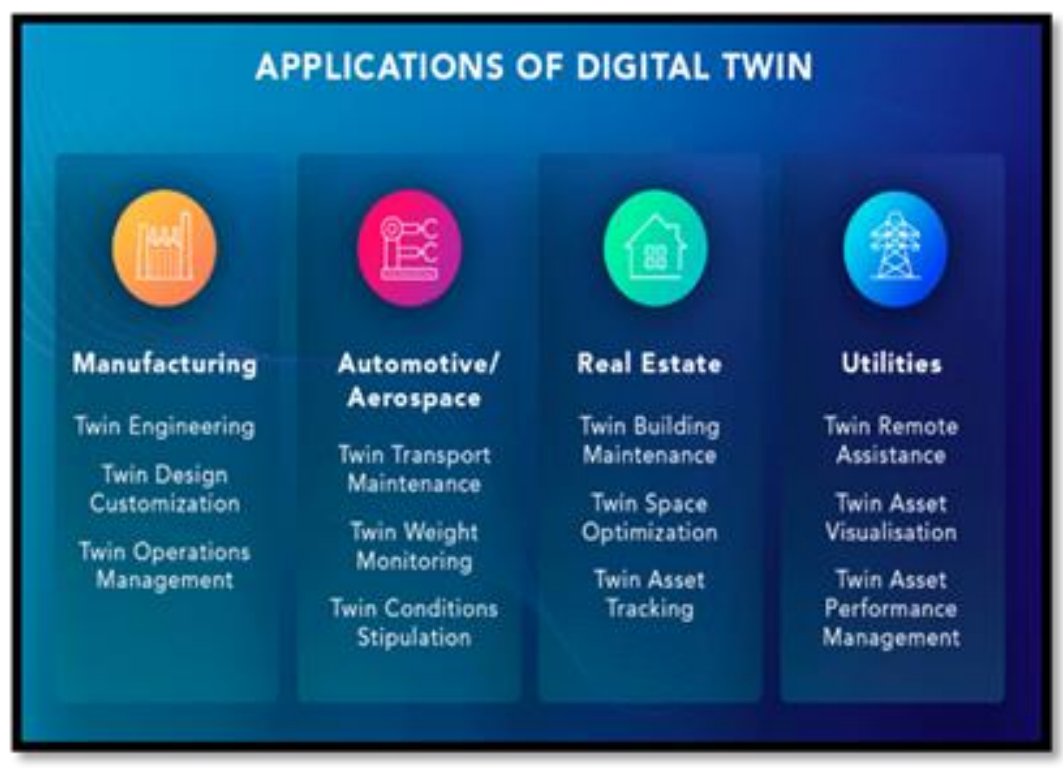

Figure 2 : Application of Digital Twins

(Source: Jones et al. 2020)

Product development, demand forecasting, assembly execution, and apparatus PHM are just a few of the applications that can benefit from the digital twin services. A series of exchanges seen between anticipated, perceived, and unique features is called a feedback loop in product design. An anticipated product in a designer's imagination is transformed into a digital representation in an interpreted world by using actual physical items as the basis. Designers must collect and analyses a large amount of data in order to gain important insights. However, product data is one of the most essential assets, and it is difficult to obtain. Designers are also unable to handle large amounts of data. Above-mentioned issues can be resolved via the use of services. Using the services management platform, designers can easily submit their requirements.

Modeling and algorithm services will be matched with the data services needed by designers. The results are returned to the designers by calling, merging, and running these services. As per the words of Pang et al. (2021), design quality and feasibility must also be verified once the product's function structure and components have been created. It is possible to accurately predict product behavior through the use of digital twins without having to wait for a product prototype. Using visual representations in product design is made simple by using services that reduces discrepancies between anticipated behavior and design behavior, reduces design cycle time, and reduces product costs. Manufacturers generally refer to the entire process, which begins with raw materials and ends with manufactured goods, as product manufacturing. It is important to plan production in advance in order to decrease costs, reduce manufacturing costs, and enhance efficiency. Planned and executed output can be improved with the help of the digital twin. 


\section{Challenges in digital twins}

While considering the positive impacts of digital twins, there are also some difficulties that the organizations need to understand to enhance the efficiency of digital twins. Here in this part of the research article a brief overview will be provided on the challenges of digital twins. Customers' expectations, product design, and processing technologies frequently cause manufacturing sectors to operate in an environment that is unpredictable and continuously changing. Due to shifting regulations and variations across borders, technology, communications, and data flow restrictions contribute to the uncertainty.

The complexity of production systems is increased by such uncertainties. As a result, more complicated systems are necessary to handle such complexity. Components, communication routes, and sophisticated information processing combine to create complex systems that are difficult to anticipate. As per the words of Wang et al. (2021), the physical items in the production environment are the focus of the conventional sequential system engineering approach. Creating a prototype was not only time-consuming and expensive because of the little margin for error, but it was also costly if it went wrong and had to be redone. With its capacity to model and simulate digitally, the DT has the potential to alter this traditional method.

\section{Methods and materials}

The study is founded on positive philosophy, and it uses positivism to analyses prior pieces of literature, such as papers, journals, and other conversations around the use of DT. According to Ladj et al. (2021), the deductive strategy of this research is also important in developing research hypotheses and objectives by deducing the theories and background study of the research. The research, on the other hand, is focused on the use of digital twins in the depiction of a real item, process, or service, and it employs a descriptive design. The study employs a secondary data gathering approach that allows the researcher to obtain trend-based patterns from publications and journals connected to DT (Lektauers et al. 2021). Secondary data analysis, on the other hand, comprises a review of existing literature to determine the picture of the issue under consideration. This study also includes a secondary data analysis that will identify themes relevant to the study's goals and analyses them for success. Since the research has followed a secondary source of data collection technique therefore, the research article has collected all the information and vulnerable data from peer journals, articles, and scholars from 2017 to 2021.

\section{Result and discussion}

As part of its analysis of the state of digital twin research, this study examined ideas, potential anticancer and industrial applications in order to determine the current state of digital twin. To improve clarity and precision, the notion should be refined using industry best practices (Park et al. 2020). Key technologies for digital twins were examined and assessed, including advanced analytics, increased modeling technologies, and model based simulation technologies. The lifetime phases of digital twin applications were outlined. Different lifespan phases were used to organize observations and future work recommendations for digital twin research. Factories now rely on one single information system to integrate data from downstream and upstream businesses such as product service. In product development, production, and service processes, digital twin technology delivers an effective solution for data management. Data from each phase of the product life cycle is accessed to combine the material flow, the information flow, and the business flow.

As per the words of Aheleroff et al. (2021), using digital twin technology, manufacturers may create a single product virtual model that incorporates lifecycle information. This programming code will be extended to include products management and servicing, as well as product recycling, in the downstream phases of production. In order to create a cloud of manufacturing-oriented digital twins by using the factory's flattened structure. In order to do diverse tasks intelligently, digital twins cloud optimization management has been unified and consolidated. In the meanwhile, business requirements, information technology, and organizational resources are being integrated and improved, allowing for harmony to be achieved across the industrial design, production, and service stages. Innovation in factories is facilitated by the information integration system. The final version of the product is described in the product design model, but there are no manufacturing requirements specified. In order to assist manufacturing operations, a digital twin of the system is vital. Product design and manufacturing processes are connected via a digital twin of the process.

In the recent year more than $75 \%$ of organizations are utilizing the digital twins system to enhance the performance of their organization. According to Barykin et al. (2021), firms that are connected with digital twins are $30 \%$ more effective than firms without digital twins. On the other hand, a series of digital twins, compromising manufacturing procedure model, process attribute information and asset models are displayed as three dimensional models throughout the production process. In order to track the efficiency and implementation conditions of multiple activities, data integration technology is being used (Tao et al. 2018). An annotation procedure digital twin's met model is created by combining the result of three algorithms such as a percolator net based model of regular engagement, platforms Simulation models linguistically knowledge sharing model and a procedure visualization model. As a result of paradigm unification and attribute mapping rules, these four models offer information for the operating digital twin met model from diverse viewpoints. 


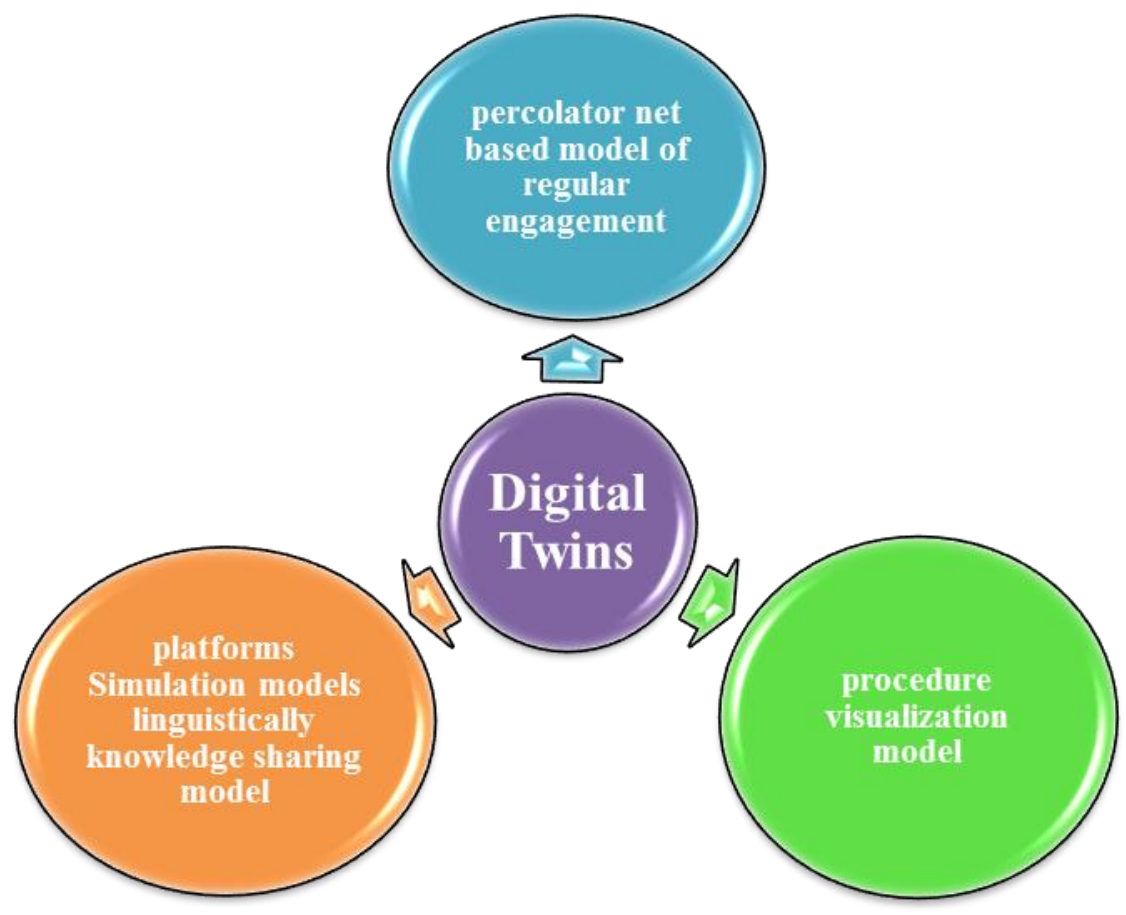

Figure 3: Three dimensions of Digital Twins

(Source: inspired by Tao et al. 2018)

Operations digital twins should be adoptable, customizable and dynamic due to the variety and dynamic nature of the interaction behaviors at the production site. A variety of operation digital twin met model can be created by setting the parameters and mapping relationships between the four models. Various processing jobs at different granularities may be simulated and monitored in this way. In the design phase, digital twins are used to optimize products and manufacturing systems iteratively, while maintaining data integrity. Virtual assessment and confirmation are the major applications. In addition to verifying that the design output is in line with the design objective, the digital twin may also be used to uncover unanticipated undesirables. When it comes to creating items, however, most publications on digital twins have been focused on checking physical objects or assessing their performance.

Digital twins have not yet been used to create a new item from scratch. Digital twin deployment is further hampered by low digital design levels in some sectors. In addition, as per the words of Schroeder et al. (2017), digital twins are also used in production in a variety of ways. Industrial systems and manufacturing processes are the primary targets of digital twins. In the workshop, less attention is being paid to the goods because information on the products' manufacturing process will be useful in the servicing phase of their life cycle. Traditional methods of manufacturing cannot enhance processing quality and cut production costs as efficiently, dynamically, or intelligently as digital twins can.

\section{Conclusion}

This research article is based on the impact of utilization of digital twins in representation of a physical object, process, or service. Through merging the cyber and physical worlds in production, the digital twin (DT) offers a potential prospect for smart manufacturing and industrial applications. DT's functionalities may be expanded via a service-oriented architecture. Development, manufacture, and PHM may all benefit from DT's capabilities. Specifications are provided for how various components of the digital twin are encapsulated and used as services. The research is in its infancy at the moment. DT simulation and service marketing approaches still need to be improved and enhanced, and more work has to be done. 


\section{Reference}

1. Aheleroff, S., Xu, X., Zhong, R.Y. and Lu, Y., 2021. Digital twin as a service (DTaaS) in industry 4.0: an architecture reference model. Advanced Engineering Informatics, 47, p.101225.

2. Barykin, S.Y., Kapustina, I.V., Sergeev, S.M., Kalinina, O.V., Vilken, V.V., de la Poza, E., Putikhin, Y.Y. and Volkova, L.V., 2021. Developing the physical distribution digital twin model within the trade network. Academy of Strategic Management Journal, 20, pp.1-18.

3. Cimino, C., Negri, E. and Fumagalli, L., 2019. Review of digital twin applications in manufacturing. Computers in Industry, 113, p.103130.

4. Jones, D., Snider, C., Nassehi, A., Yon, J. and Hicks, B., 2020. Characterising the Digital Twin: A systematic literature review. CIRP Journal of Manufacturing Science and Technology, 29, pp.36-52.

5. Ladj, A., Wang, Z., Meski, O., Belkadi, F., Ritou, M. and Da Cunha, C., 2021. A knowledge-based Digital Shadow for machining industry in a Digital Twin perspective. Journal of Manufacturing Systems, 58, pp.168-179.

6. Lektauers, A.R.N.I.S., Pecerska, J.E.L.E.N.A., Bolsakovs, V.I.T.A.L.I.J.S., Romanovs, A.N.D.R.E.J.S., Grabis, J.A.N.I.S. and TEILANS, A., 2021. A multi-model approach for simulation-based digital twin in resilient services. WSEAS Trans. Syst. Control, 16, pp.133-145.

7. Pang, T.Y., Pelaez Restrepo, J.D., Cheng, C.T., Yasin, A., Lim, H. and Miletic, M., 2021. Developing a digital twin and digital thread framework for an 'Industry 4.0'Shipyard. Applied Sciences, 11(3), p.1097.

8. Park, K.T., Son, Y.H. and Noh, S.D., 2020. The architectural framework of a cyber physical logistics system for digital-twin-based supply chain control. International Journal of Production Research, pp.1-22.

9. Qi, Q., Tao, F., Zuo, Y. and Zhao, D., 2018. Digital twin service towards smart manufacturing. Procedia Cirp, 72, pp.237-242.

10. Schroeder, G.N., Steinmetz, C., Pereira, C.E. and Espindola, D.B., 2017. Digital twin data modeling with automationml and a communication methodology for data exchange. IFAC-PapersOnLine, 49(30), pp.12-17.

11. Tao, F., Zhang, H., Liu, A. and Nee, A.Y., 2018. Digital twin in industry: State-of-the-art. IEEE Transactions on Industrial Informatics, 15(4), pp.2405-2415.

12. Wang, X., Wang, Y., Tao, F. and Liu, A., 2021. New paradigm of data-driven smart customisation through digital twin. Journal of manufacturing systems, $58, \mathrm{pp} .270-280$. 